\title{
Improving the evaluation of generator matrix $G$ by initial upper bound estimation
}

\begin{abstract}
Space-Time Trellis Code (STTC) can achieve both the diversity and coding gains. To maximize the advantages of STTC, two design criteria for slow Rayleigh fading channels will be used: i.e. the rank and determinant criteria. This paper focuses on the determinant criteria, which involves the evaluation of the generator matrix G. Evaluation is improved by pruning the search process earlier, which is made possible by estimating the initial upper bound prior to the search. In order to reduce the search complexity, the initial upper bound will be calculated at the minimal cycle. Comparatively, it can reduce the search space by $25.8 \%$.
\end{abstract}

Keyword: Generator matrix G; Minimum determinant; Minimal cycle; Initial upper bound; Rank and determinant criteria 